\title{
What is Heat; the Photon is Heat
}

\author{
Bhekuzulu Khumalo \\ 305 - 19 Northern Heights Drive, Richmondhill, Ontario, Canada \\ bhekuzulu.khumalo@gmail.com
}

\begin{abstract}
All photons will burn you. How do we describe photons as having different amounts of energy? This paper illustrates photons do not have different amounts of energies, rather different types of energy. The experiment of 1800 provides enough data to be analyzed because it has that third point to question the idea that photons carry different amounts of energy. This paper argues that all photons have equal amounts of energy just different types of energy. The composition of the energy within a photon depends on the frequency of a photon, a lower frequency photon like those represented by infrared can boil water faster than higher frequency blue light. A higher frequency photon like a gamma particle is stopped by lead. Given the nature that heat is from photons we can start thinking of sophisticated thermometers that give us the quality of heat not just the quantity of heat. It is the atmosphere that gives more evidence around the nature of photons, we can understand the cycle of the photon/ photonic cycle/ electromagnetic cycle, allowing us to ponder on deep philosophical meanings, intelligent life is there for universe to sustain itself, as well as ask the question why we are not burning given the nature of low frequency electromagnetic radiation. And for those vigorously looking for habitable planets out there, the idea of the circumstellar habitable zone must change to accommodate the proper understanding of heat.
\end{abstract}

Keywords: circumstellar habitable zone, electromagnetic spectrum, heat, photon, photonic cycle, photonic energy, photonic mist, quality of heat, quantity of heat, sophisticated thermometer

\section{Importance of Works}

This paper challenges the majority of what is accepted as heat, how hot is something. Take what is considered as an authoritative introduction to heat from ${ }^{1}$ www.physicsclassroom.com, here is how they define heat:

- The degree of hotness or coldness of a body or environment.

- A measure of the warmth or coldness of an object or substance with reference to some standard value.

- A measure of the average kinetic energy of the particles in a sample of matter, expressed in terms of units or degrees designated on a standard scale.

- A measure of the ability of a substance, or more generally of any physical system, to transfer heat energy to another physical system.

- Any of various standardized numerical measures of this ability, such as the Kelvin, Fahrenheit, and Celsius scale

Note they cannot tell us what is hot or what is cold, just because something is cold to you does not mean it is cold to a rock, just because something is hot to you does not mean it is hot to the sun. Very personal definition don't forget everything is just information, one might ask why compare a human to a rock, the answer is because it is all ${ }^{2}$ information. 
A well known teaching academy, the ${ }^{3}$ Khan Academy, rumoured to be friends with the likes Bill Gates, their definition of heat is:

- Heat, $q$ is thermal energy transferred from a hotter system to a cooler system that are in contact.

- Temperature is a measure of the average kinetic energy of the atoms or molecules in the system.

- The zeroth law of thermodynamics says that no heat is transferred between two objects in thermal equilibrium; therefore, they are the same temperature.

- We can calculate the heat released or absorbed using the specific heat capacity $C$, the mass of the substance $m$, and the change in temperature $\Delta T \backslash$ Delta $\backslash$ text $T \Delta T, T$ in the equation:

$q=m \times C \times \Delta T$

This still does not tell us what heat is what is being transferred from this hotter system to cooler system, then why is all the heat not escaping the earth, it is surrounded by cold space, hopefully by the end of this paper a teacher will be to understand what temperature is, and what they are calculating when they say they can calculate heat released and absorbed and teach the reality. To appreciate heat is to appreciate the electromagnetic workings.

That is the importance of this paper, because it allows us to look at heat from the quantum, where it originates, it rejects most of the canonized knowledge surrounding heat and gives what is a comprehensive reasoning for rejecting that knowledge as it can not be true and thus is not information.

\section{Introduction}

In the paper, ${ }^{4}$ "heat, quantum mechanics, and information" the idea of a particle that is nothing, but pure heat was speculated upon. An experiment was provided, at the end of all talk only observation, an experiment can verify scientific thought no matter how sound the mathematics and logic is. What if the idea of the pure heat particle is as correct as can be but the author of the mentioned paper arrived at the thought with an incorrect first premise.

To appreciate the rest of this paper an illusion must first be understood concerning light, how hot something is, and the mind. Imagine being inside where the temperature is $27^{\circ \mathrm{C}}$. and outside it is $-10^{\circ \mathrm{C}}$ and outside it is bright, there are no clouds. If one goes outside in a t-shirt and stand by the door, the heat from inside will protect one from feeling the full effect of the cold. The first time this happens, and it is bright outside, standing by the door, one would think that the heat is coming from the sun. The reason is we have associated a bright sun shiny day with warmth. A little further away from the door and the deadly reality of the cold will bite your skin with all that bright sunlight.

It is important to appreciate the above illusion because the concept of heat is not everything we have thought it to be. A simple experiment might be able to change our view of what is heat, understand it to as much depth as possible. This experiment has been repeated thousands of times. It is an experiment first carried out in 1800 by William Herschel, much simpler than a collider, but perhaps it can give us insights that colliders, magnets, and sophisticated machinery have failed to give. The experiment clarifies ideas that truly needed an experiment, even though it challenges the idea of a single heat particle.

We have much knowledge concerning the photon, one can search engine ${ }^{5}$ electro magnetic wave and they receive many websites detailing the types of photons and the amount of energy each particle possesses to every decimal detail. We know the electromagnetic spectrum is illustrated as on one side low frequency, longer wavelength, and lower photon energy to high frequency, shorter wavelengths and higher photon energy. 


\section{What do we Mean by Energy}

Energy is one of the most confusing concepts. Accepted theory says that photons carry within themselves different amount of energy, that is why it is always illustrated from what is low energy to high energy. There is no point in discussing energy if we do not go to those that have defined energy. Understanding information, one will understand that there is not one single piece of information with greater value than another piece of information in this universe, otherwise the universe could not exist, but, we recognize critical points in how knowledge operates, what makes us understand more. Knowledge is a human phenomenon, information is the universe, knowledge is just information that we understand. Whatever we consider as energy, the equation from Einstein that is a critical point in our understanding of the universe, $\mathrm{E}=\mathrm{MC}^{2}$ can not be broken, because if it is wrong about the photon it is worth nothing. We as scientists have accepted Einstein gave us the standard definition of what energy is.

How can 2 photons have different amounts of energy given that they both have the same mass and are not at rest, what does one mean when they say that a gamma particle has more energy than a radio particle given that both are essentially photons. It is false not intentionally but because there is some other information that was missing that will reconcile the reality that photons must have the same amount of energy, and then we understand quality of information.

Perhaps understanding that energy is ability to do work we can ask ourselves a simple question like if gamma particles have more energy than radio particles, how is it they are more able to do more work? It is the misunderstanding of what energy is that we do not get the full picture of the energies involved in the electromagnetic spectrum.

Before we confuse ourselves in philosophical debates we must use a dictionary and get a definition of knowledge, knowledge being nothing more than facts we know. The internet dictionary www.dictionary.com defines energy as:

1. the capacity for vigorous activity; available power: I eat chocolate to get quick energy.

2. an adequate or abundant amount of such power: I seem to have no energy these days.

3. often energies. a feeling of tension caused or seeming to be caused by an excess of such power: to work off one's energies at tennis.

4. an exertion of such power: She plays tennis with great energy.

5. the habit of vigorous activity; vigor as a characteristic: Foreigners both admire and laugh at American energy.

6. the ability to act, lead others, effect, etc., forcefully.

7. forcefulness of expression: a writing style abounding with energy.

8. Physics. the capacity to do work; the property of a system that diminishes when the system does work on any other system, by an amount equal to the work so done; potential energy. Symbol: $E$

9. any source of usable power, as fossil fuel, electricity, or solar radiation.

As we can see the common theme in all the variations of energy is basically the first definition, "the capacity for vigorous activity, available power." The capacity for vigorous activity being the ability to do work, and available power being the potential to do that work, or what is considered potential energy. 
The new is always fascinating, especially more than something one has been around seemingly forever. It does not take an enormous amount of energy to start what could eventually be a forest fire, a cigarette butt. The energy within a cigarette butt will never light coal, let alone equal what is needed to start a nuclear reaction. But it is enough to give one a forest fire, forests fires can reach very high temperatures, they are fairly hot, that heat being radiation, most accept more infrared radiation than what we would consider usual. The amount of heat being generated is that not energy, the ability to do work? Medium rare steak is better than one that is not cooked.

\section{The Quality of Heat: Herschel Experiment}

In 1800 William Herschel discovered infrared by using a simple experiment. He measured the temperature of light when it is split into its different constituencies. The experiment can be found on the site ${ }^{6} \mathrm{Cool}$ Cosmos, a NASA education and outreach website. They should be reliable. What does this experiment mean with the knowledge and information we posses in the $21^{\text {st }}$ century. It must be noted that our argument is that all massless particles have the same amount of energy, it all depends on what one is looking for. Gamma particles are extremely penetrating, but neutrinos go through the earth, they are moving, that takes energy, another bigger topic.

The ${ }^{7}$ experiment is laid out nicely, 3 thermometers taking the temperatures of different colored light. In the experiment set out in above website, all three thermometers where checked all read the same temperature $76^{\circ} \mathrm{F}$ in the shade. They where placed in the spectrum and after 1 minute there was a marked difference the final readings where:

blue $80^{\circ} \mathrm{F} \quad$ yellow $83^{\circ} \mathrm{F} \quad$ red $86^{\circ} \mathrm{F}$ not forgetting outside spectrum it was $76^{\circ} \mathrm{F}$

\begin{tabular}{|l|l|}
\hline type of light & temp $^{\circ} \mathbf{F}$ \\
\hline shade & 76 \\
\hline blue & $\mathbf{8 0}$ \\
\hline yellow & $\mathbf{8 3}$ \\
\hline red & $\mathbf{8 6}$ \\
\hline table:1 results of Herschel experiment & \\
\hline $\begin{array}{l}\text { source: } \\
\text { http://coolcosmos.ipac.caltech.edu/cosmic_classroom/classroom_activities/herschel_example.html }\end{array}$ & \\
\hline
\end{tabular}

As can be seen from table 1 we have a distribution range, amongst the colors it is fairly linear as expected. For that we must return to the logic that was found in the paper, "heat, quantum mechanics, and information", that was merely using logic. It was speculated in the paper that there must be a particle of pure heat at the lowest frequency end, and an experiment was provided that needs to occur at absolute zero. The failure of this paper, as all logic ultimately will without experimentation one way or another, is that it contributed all heat to this particle, no with experimentation we understand that it is the particle with the most heat, all photons have heat.

How does Herschel's experiment support the idea of a tshison, not only does it support the idea of a tshison, but we can finally reconcile what has appeared as differences in energy that each class of photon possesses. Figure 1 illustrates Herschel's experiment in terms of frequency of temperatures. 


\section{Figure 1: Herschel's Experiment Frequency}

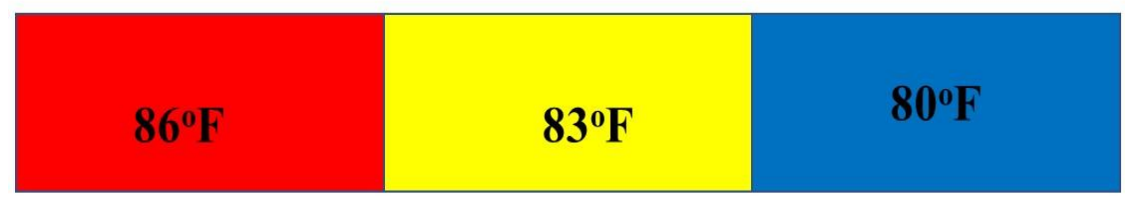

Increasing frequency

There can be no argument that as the frequency rises the temperature is dropping, and we can see it is dropping in a linear fashion, in a simple $Y=X$ fashion not $Y=a X+n$. The variable $n$ cannot exist, if there is no electromagnetic activity, the temperature is not $n$, it is zero. But, we know that photons with higher frequencies have greater penetrating power and for all purposes have considered this to mean more energy, then why does red light produce more heat.

\section{The Energy of a Photon}

Could this not be an illusion of a complete picture of the energy that a particle possesses, but the red light will make the water boil quicker, how can that not be energy work is being done, the water is heating up quicker. It has to be our understanding of what energy is that is confusing us, but once we accept that boiling water quicker means use of energy, then we can reconcile the 2 different energies as illustrated in figure 2.

\section{Figure 2: Energy Distribution of Photons}

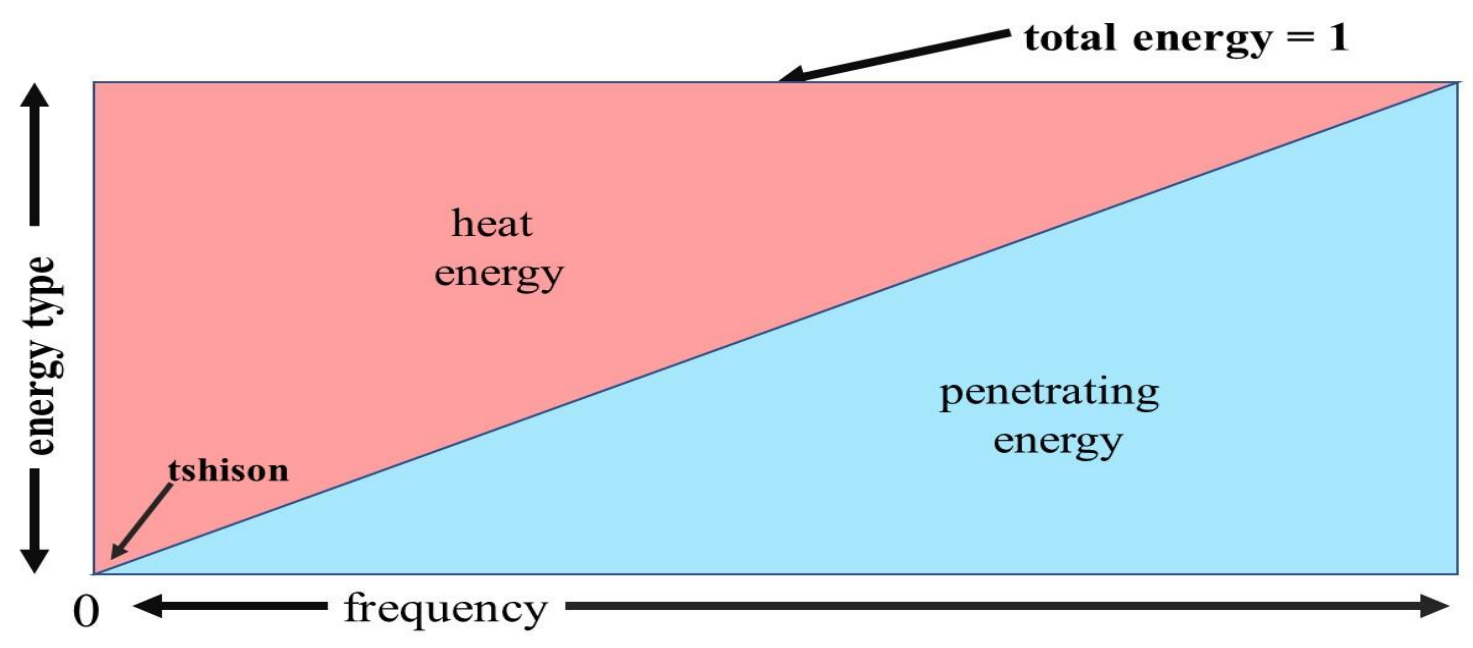

One can with confidence finally draw up figure 2, illustrating that all photons have the same amount of energy, there is trade of between heat and penetrating energy both being a fraction of the whole, and the whole is 1 , somebody must think of a name for this 1,1 what, but it is 1 so that people can understand these are just fractions of a whole. We can postulate equation $1^{\prime \prime}$

(1) $\mathrm{Ep}=\mathrm{He}+\mathrm{Pe}$ where

$E p=$ energy of a photon

$\mathrm{He}=$ heat energy 
$\mathrm{Pe}=$ penetrating energy

From here we can derive equation 1.1

(1.1) $\mathrm{He}+\mathrm{Pe}=f($ freq $)$ where

freq is frequency

From equation 1 we also get 1.2

(1.2) $\mathrm{He}+\mathrm{Pe}=1$ and:

One will be defined in time, but we already have a clue, highest frequency of gamma approaches 1 in penetrating energy and the lowest frequency approaches 1 but mostly heat energy (we will further understand, after all this paper is to highlight what was canonized as wrong). It is all a ratio, a trade off. Remember when using units it is arbirtrary in many sense, one could easily define $\mathrm{He}+\mathrm{Pe}=10$ or 100 , but understanding it is photons we can get to the actual value of $\mathrm{He}+\mathrm{Pe}$, of what this truly is.

Given figure 1 and figure 2, we must understand figure 3. Figure 3 is the same as figure 1 , except it includes the temperature of the shade.

\section{Figure 3: Herschel's Experiment Frequency 2}

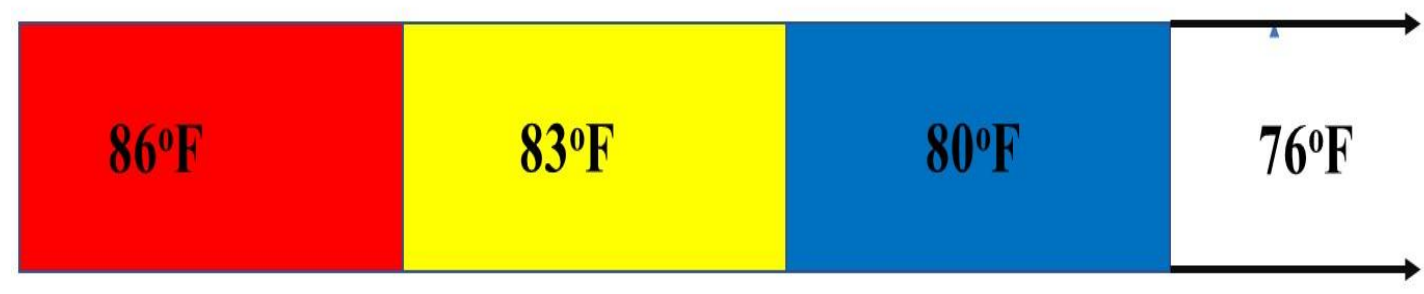

\section{Increasing frequency}

We must answer for the lower temperature in the shade, why seemingly off linear. In the shade we see that the temperatures are significantly lower than when visible light is split into it's constituencies. All colours of the prism break down, red, orange, yellow, green, blue exhibit temperatures significantly higher than the background. Couldn't one put the prefix infra on all visible light, a philosophical debate. What is off interest to us is why the background temperature is much lower than say the blue, or infra blue if one wanted, how would they be logically wrong?

\section{How hot is it?}

The only answer possible for the question why the control temperature is lower is that in the atmosphere there are many different types of photons with different frequencies and the temperature is merely a strict average of their energy types. If one thinks about it, visible light can never ever be the dominant photon on this earth, that is merely an illusion because that is what we see. But that red light 20 feet away from you might get to you eye uninterrupted thanks to photons not interfering with each other, or those many colors from the television, but remember in those $20 \mathrm{ft}$, and in between the screen tv and yourself there is enormous amount of photon activity, many different types, far more than the visible light that is being radiated to the eyes. As every photon is a mixture of both heat and penetrating energy, the average temperature is reduced to show the average heat being generated. 
From figure 4 we can understand the temperature of an area, remember, thermometers take temperatures of an area, it is affected by trillions of photons.

\section{Figure 4: How Hot is It?}

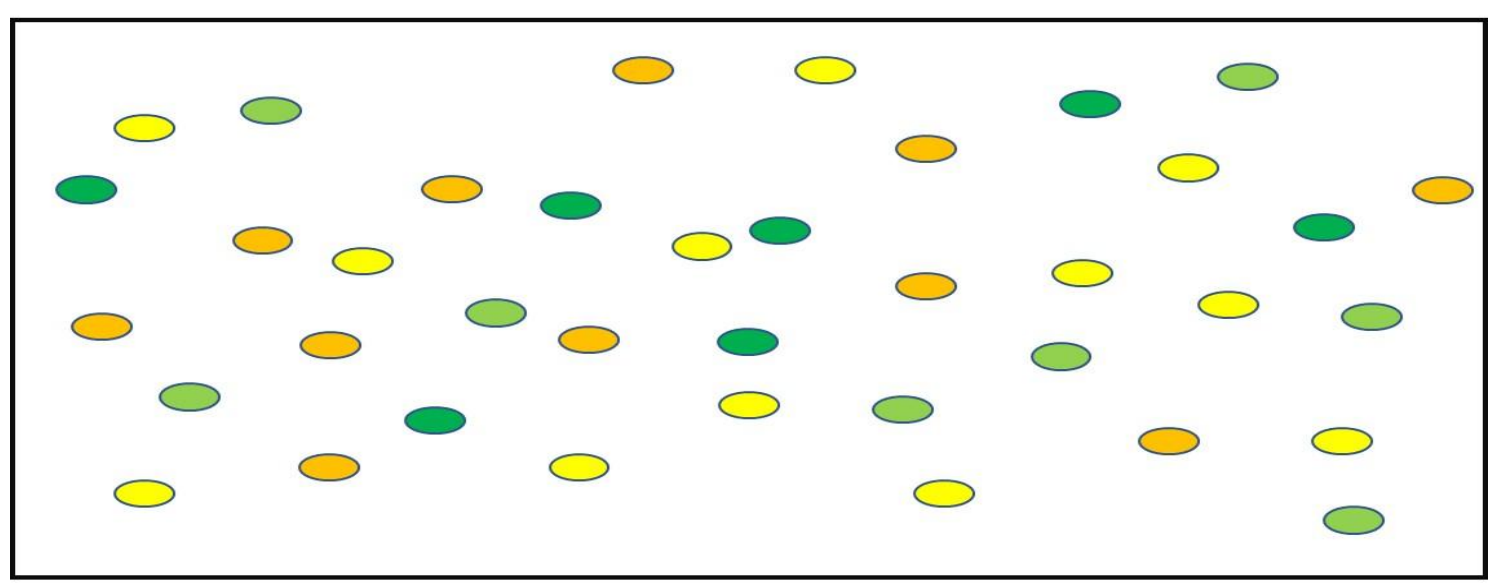

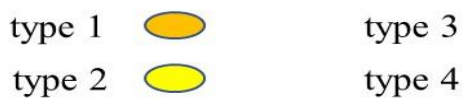

The temperature of the area represented in figure 4 is the weighted average heat energy of the 4 types of photons represented. We get equation 2 .

(2) $\mathrm{T}=\sum$ Hetype $_{\mathrm{i}} \quad$ Where:

$\mathrm{T}=$ temperature

$\mathrm{He}=$ heat energy

type $_{\mathrm{i}}=$ class of photon

Each class of photons heat contribution is a simple sum of each individual photon being measured by the scale, in most cases a mercury thermometer.

Going back to Herschel's experiment, when we measure yellow, blue and red light, that light has been separated by man, and this separation shows the individual contributions of heat per light band to the entire heat that we feel in the atmosphere. However, as we can see from figure 3, the average heat in the atmosphere is less than the visible light. There is a complex mixture of photons in the atmosphere, and from the Herschel experiment we can conclude they are of higher frequencies is what causes us to achieve temperatures that are comfortable to us. Logical conclusion, too much visible light means greater heat.

Given equation 1 above and figure 4, we can understand what type ${ }_{i}^{\prime}$ s contribution is.

(2) $\mathrm{T}=\sum$ Hetype $_{\mathrm{i}}$

(3) $\sum$ Hetypei $=$ Hetypei $X \mathrm{~N}=$ NHetypei where:

$\mathrm{N}=$ number of type $_{i}$ photons. 
This should be easily understood say all photons with a certain frequency have the same composition of energy types and thus same amount of heat energy and penetrating energy when satisfying $\mathrm{He}+\mathrm{Pe}=1$.

Thus when we look at figure 4, if we know the number of photons we should not even need a thermometer to measure the temperature given equation 2 . The temperature represented by figure 4 is:

$T=$ Hetype $_{1} \times N_{1}+$ Hetype $_{2} \times N_{2}+$ Hetype $_{3} \times N_{3}+$ Hetype $_{4} \times N_{4}$ where

$\mathrm{N}_{\mathrm{i}}=$ number of $\mathrm{i}$ type photons.

For those familiar with the nuclear industry, including nuclear power generation, especially in North America, they go through awareness sessions of the dangers of radiation, pictures are shown with radiation burns, how they can start as blisters and continue to burn on, burns they are even though they be coming from gamma radiation. All photons will burn in enough concentration, but the burn from gamma is not hot.

Equation 1.2 gave us:

$\mathrm{He}+\mathrm{Pe}=1$

What is known as photon energy has been termed Pe. We know from text books that what has been termed as $\mathrm{Pe}$, penetrating energy, has been for some decades known as photon energy. This paper has argued that is only part of the energy a photon has in most circumstances. Pe as from book is Planck constant multiply the speed of light divided by the wavelength, or

$E=h c / \lambda$ where:

$E=$ photonic energy

$\mathrm{h}=$ plancks constant

$c=$ speed of light

$\lambda=$ wave length.

As $c / \lambda=f$ where

$f=$ frequency Thus

$E=h f$

Understanding the relationship from equation 1.2. We can express figure $1.2 \mathrm{~A}$ :

$\mathrm{He}+\mathrm{Pe}=\mp$ where:

$F$ is the total energy of the photon and is the same for all photons. Thus:

$\mathrm{He}=\mp-\mathrm{hf}$

It is time to give heat energy from a photon a name, it shall be named Tshisa, from the hypothesised heat particle, tshison. 
From equation 4 we understand that one limit is when $F=h f$, that means theoretically such a photon has not heat energy, it would register zero on the tshisa scale. The other limit is when $\mathrm{hf}=0$. That particle theoretically is just composed of heat energy, obviously this is not possible, but the first electromagnetic phenomenon that happens after $\mathrm{hf}=0$ is the tshison, the longest wavelength, most easily absorbed of the photon particles, and carries the highest heat signature, highest tshisa reading on the heat scale.

Given equation 4, we can allow modifications to figure 2 and get figure 4.2.

\section{Figure 4.2: Heat and "photon" energy}

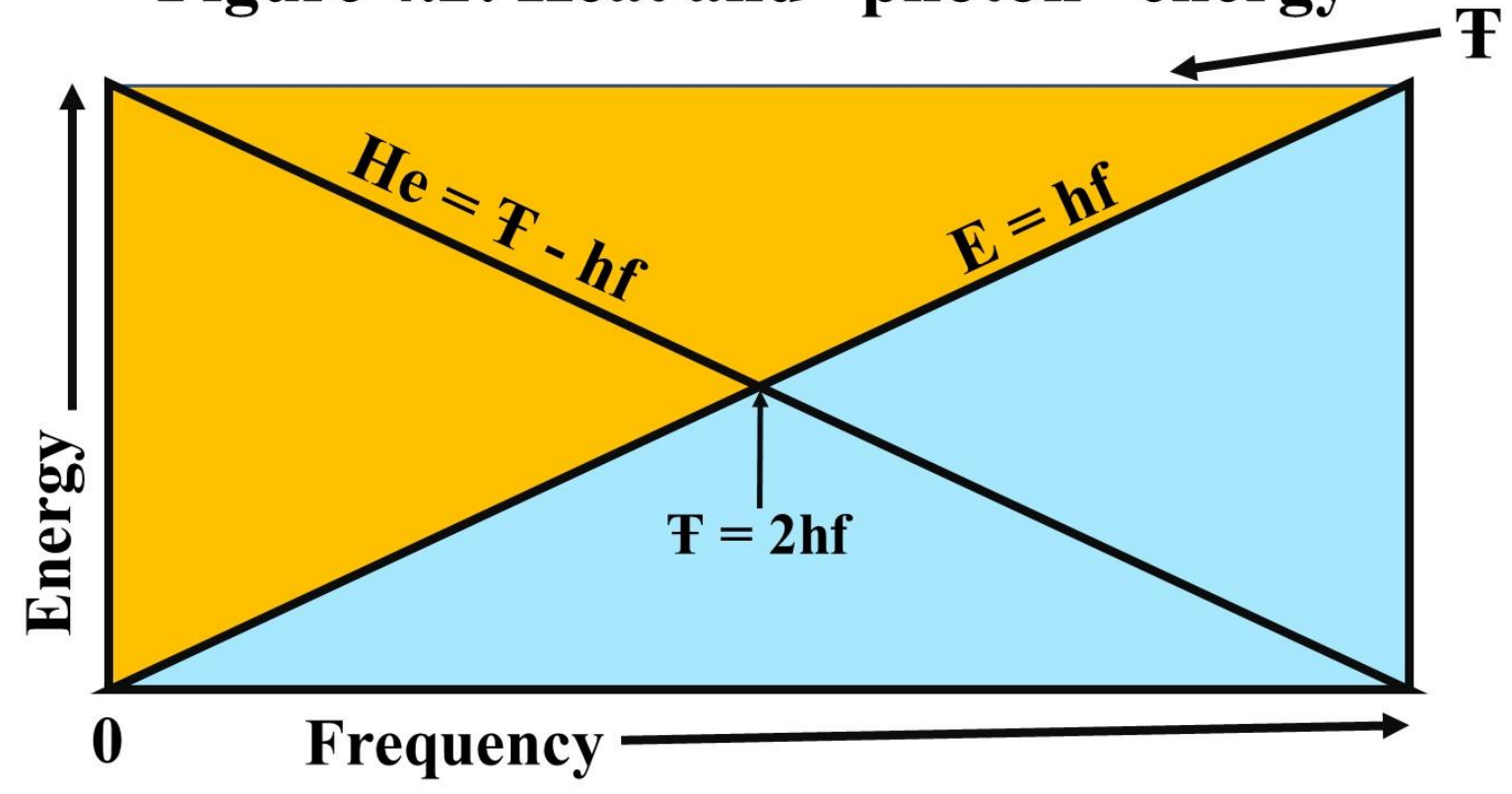

\subsection{When is an equation less than a sequence?}

When we look at figure 4.2 we notice that when a photon has equal amounts of the types of energy we get a situation represented by equation 5 :

$$
F=2 h f(5)
$$

At first glance putting forward an equation like equation 5 seems like a so what, so $\mp=2 \mathrm{hf}$, we can plot it like any equation, it can be plotted from zero to infinity. It is true that can be done but it would be false, because the scale represented by equation 5 is a point. To chart it out is meaningless though obviously one can.

The only way one can imagine equation 5 as a continuous function is to see figure 4.2 in 3 dimensions instead of 2, but it still will not make sense because we will be seeing theoretically possible representations of photonic energy that do not exist, an infinite different expressions of figure 4.2 but each $\mp=2 \mathrm{hf}$ different from the last even though there is only one true $F=2 \mathrm{hf}$, the one that is represented in our reality.

Thus, it seems it is possible to chart out a relationship when in reality only one point between zero and infinity can ever be true. By mistake one could assume proof of a multi dimensional world mathematically. Think about it, to plot $F=2$ hf given above figure can only mean you can plot it in a 3-dimensional frame. But if you think of it as a multiverse, it means each $\mp=2 \mathrm{hf}$ is slightly different in total energy to the next, meaning total energy of photons is slightly different in the next universes, meaning total energy of each universe decreases one way and increases one way from our own universe, but one should be very weary of idea of multiverses, it's just maths. 


\section{Some Thoughts for the skeptics}

It might be difficult for one to understand that when they hold a burning stick what is burning them and what is making the stick hot is photons, radiation, a well controlled chain reaction releasing low frequency photons. Given the reality of a stick burning, what does the Khan's definition of heat mean, what does "Heat, $q$ is thermal energy transferred from a hotter system to a cooler system that are in contact" mean. What is this thermal energy that is transferred from a hotter system to a cooler system. Where is this hotter system getting the heat from, where is the stick getting heat from, why does cold air rush in, in some climates, we see cooler system contributing to hotter systems, a definition must always be constant. Heat is a result of the release of photons.

When one says temperature is kinetic energy of atoms, why are winds in the artic so called, those gusts are called gusts for a reason, and where are these atoms going inside a piece of metal that they have so much kinetic energy? Let us take a brick of gold, we see it shining yellow, it is stable at room temperature. That we can see it means there is electromagnetic activity, it is radiating light or how can we see it? When the gold brick is put in a furnace it's radiation, particularly the radiation associated with heat intensifies. The reason for this is simple. Photons are energy, we see the gold brick because of the correction of an imbalance within gold in the environment it then radiates photons to maintain that energy balance. It works the same with all photons.

That this process works the same with all photons not just visible light. Remember visible light cannot be the majority of photons in our environment, otherwise the shade temperature in Herschel's experiment wouldn't be lower than the visible light. However low penetrating photons are not as many otherwise the environment would be much hotter. Low penetrating photons as they do not pass, easily deliver heat energy at touch. In a furnace, heat energy, low penetrating photons are abundant, those are the type of photons that will be absorbed and to create an internal balance, photons of similar type will be released as they represent a similar type of electromagnetic radiation, just as when it radiates visible light.

The rigid structure of metals allows them to absorb more heat than most other materials still keeping their shape more or less, at 500 degrees for 3 days the human body would have been burnt to crisp, but the metal will keep its shape. However, as temperatures increase, to maintain a balance of photons that it receives and to balance it must radiate out what it receives. When the photon absorption and radiating of these particular type of photons, because of the quality of their energy, at a discrete number of photons being released the metal will begin to be a liquid. Its change of state allowing a greater surface area in which to allow more low frequency photon absorption and radiating.

A change of state of matter is due to intensity of the balancing mechanism within the electromagnetic spectrum. When water reaches 100 degree Celsius, it turns to steam. Appreciating the photon for what it is we can understand what is happening. Lower frequency photons are absorbed and radiated by water, thus increasing the temperature of the water, the photons being radiated affect other atoms, we know this because we have been carrying out an experiment since we started boiling water. Radiation has no direction, a lot of it is internal, hence when those low frequency photons are released the internal radiation forces increased electromagnetic activity within the next atoms ease of the increased activity internally depending on the structure of the atoms and molecules. This is illustrated in figure 5. 


\section{Figure 5: radiation movement in material}

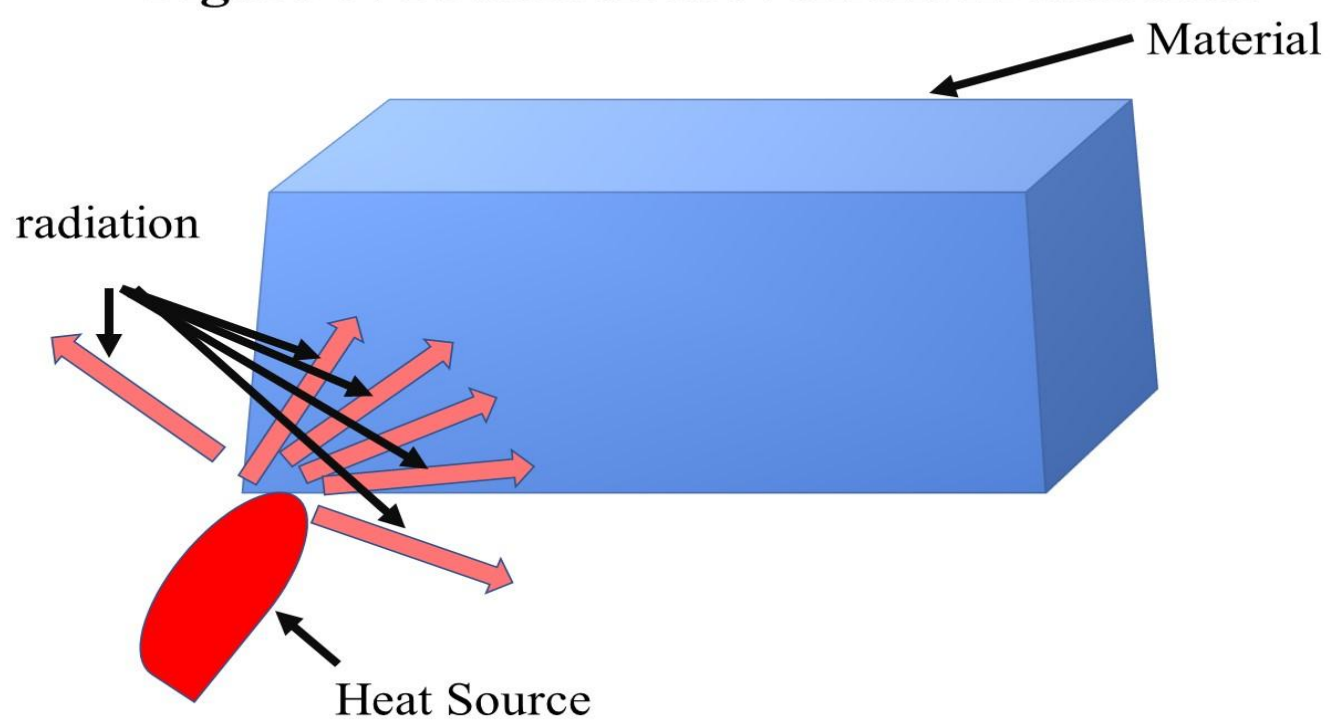

Figure 5, shows a heat source and a material. When the low frequency photons easily absorbed by the material receive heat and that causes them due to balancing of energy to radiate heat, internally and externally, this heat is all due to electromagnetism. What we sense as the material getting hotter is not the movement of atoms but the increase in electromagnetic activity involving low frequency photons.

\section{Figure 6: Electromagnetic Workings}

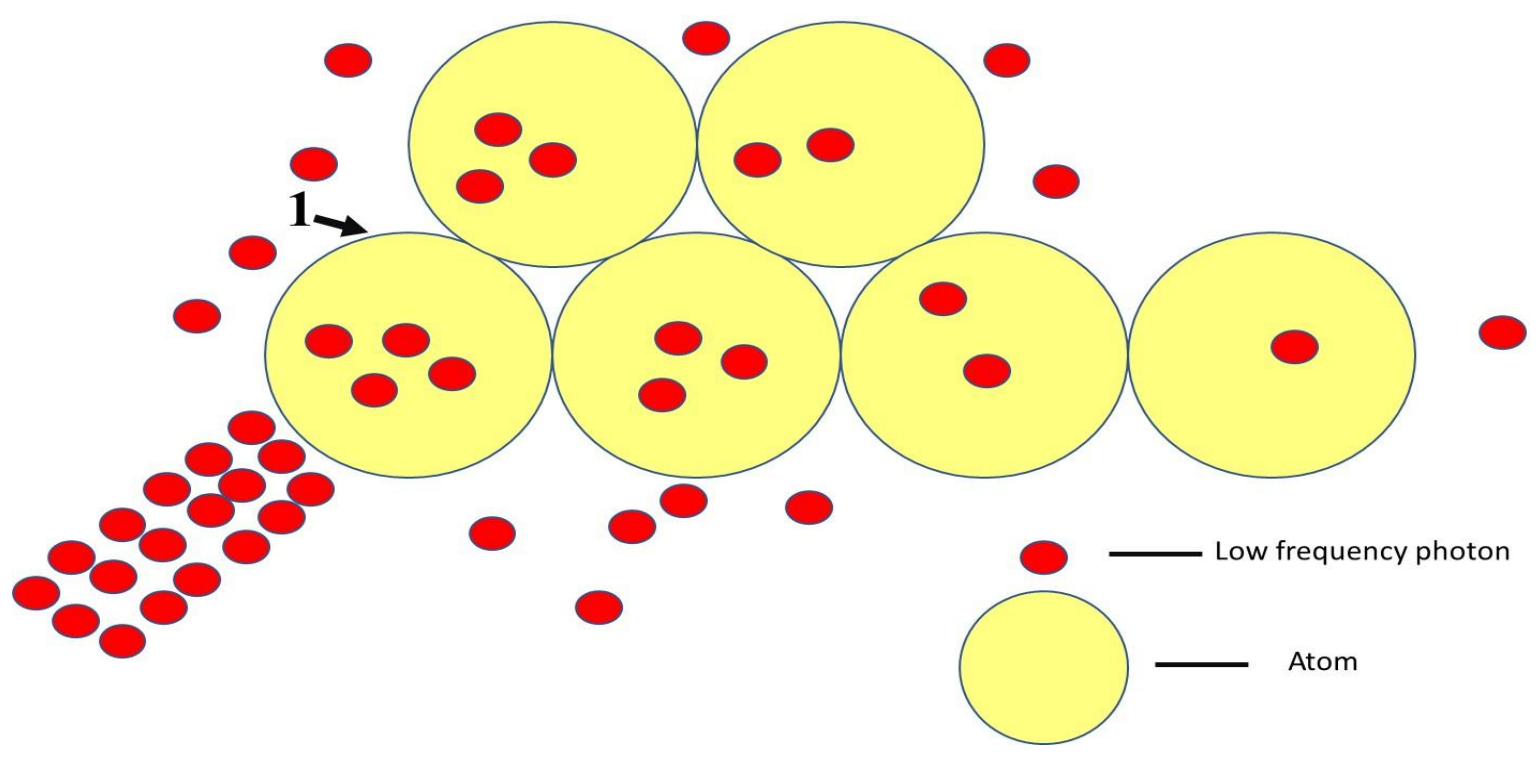

In figure 6 we see a very simplified illustration of what is going on when low frequency photons are added into the electromagnetic spectrum activity. Atom number 1 receives a new burst of low frequency atoms possibly from fire. To maintain a balance photon 1 must radiate these low frequency atoms in an exchange to maintain internal balance. It radiates photons to the surrounding area as well as to neighboring photons that in turn also radiate photons to the surrounding area as well as internally.

This is what is happening when water is being boiled, but there is a certain point, a critical point where the number of low frequency photons in the system are just to great for that state that matter changes its form, water will boil at $100^{\circ} \mathrm{C}$, what really is happening is that $100^{\circ} \mathrm{C}$ represents a certain amount of low frequency 
photons in the system that when we measure with a thermometer we see such a temperature. At this level of photons in the system, to increase efficiency of getting rid of the photons the system transforms, water boils and becomes water vapour. At a level when the amount of photons in gold reach what we have defined as degrees Celsius reaches $1064^{\circ} \mathrm{C}$, it becomes too much for gold to continue trying to balance itself that it must change form to a liquid, it can flow unless of course it is contained, flowing increases its surface area.

What about body heat that is a chemical reaction what has it to do with photons. The chemical reaction is in order to release low frequency photons that we all radiate, and we all radiate it at such a rate that we are all at the same constant release of this photons per unit of cells that we all are at he same temperature always or we are malfunctioning.

When we look at figure 6 we understand how busy it is at the atomic level, an atom will be absorbing and radiating different frequency electromagnetic phenomenon all at nearly the same time, staggering amounts of action in any time frame, be it minutes, seconds, or nanoseconds.

\section{Does a photon have rest mass?}

As all heat comes from a photon, an atom as such can never display temperature, protons, neutrons, and electrons have no temperature as such to talk about, they are neither hot nor cold, their energy has nothing to do with that. When water is boiling, the heat is from photons, the 2 particles of hydrogen and one of oxygen are themselves neither hot nor cold, those are meaningless descriptions of them. It is the quality of photons that there is temperature. Thermometers are just scales measuring how many photons are giving out heat, a thermometer only gives quantity, it is not a qualitative measuring instrument that will tell the composition of the photons that make up the heat in that location.

If we take a brick of metal and measure how much it weighs, we understand it mass. If we then heat that brick, freeze it in nitrogen, it should weigh slightly more for a few seconds as electromagnetic activity is taken to as low as we possibly can.

\section{Sophisticated Thermometers}

To get an accurate picture of how hot something is and why, sophisticated thermometers are needed. This is illustrated in figure 7.

\section{Figure 7: Sophisticated Thermometer}

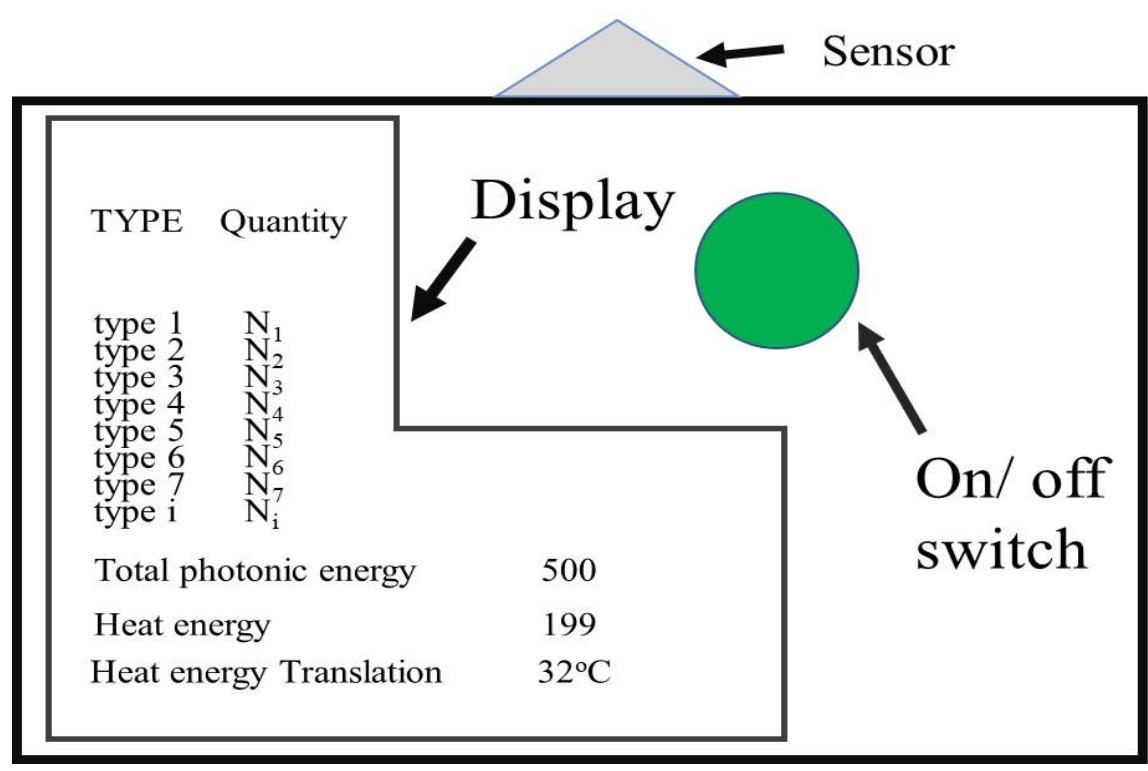


Looking at figure 7 we have an instrument. This instrument has a sensor. The sensor is attached to internal instrumentation that analyzes photonic activity. It then gives a read out on a display. The display tells us the quantity of different electromagnetic phenomenon, the total photonic energy, the total heat energy, and this is translated to give us a temperature reading we are used to, in figure 7 it says $32^{\circ} \mathrm{C}$.

The sophisticated thermometer would have a great advantage over a normal thermometer in that it not only gives quantity but quality. Two locations separated by 1000 miles can both be around $32^{\circ} \mathrm{C}$ but the energy composition significantly different given atmospheric conditions we have not yet looked at, the composition of electromagnetic phenomenon. Not only will this help climatologists but in home comfort, imagine a tinted window that knows exactly what bad photons to try and filter, changing the tint according to electromagnetic activity passing through.

The ability of measuring temperature in such a way will lead to other technologies. Having the ability to analyze as much electromagnetic phenomenon at the same time will lead to more sophisticated instrumentation on detecting phenomenon that is moving in space, natural and artificial, everything will leave behind a pattern affecting the electromagnetic spectrum if it exists. More importantly a sophisticated temperature can tell us the conditions of living things, depending on conditions life will be releasing different electromagnetic phenomenon. But first the thermometer is needed so that we understand heat.

The thermometer to be a wonderful piece of technology must accurately measure temperature merely from the electromagnetic phenomenon in the atmosphere.

\section{Herschel's experiment and Black Body Radiation}

What we do know from Herschel's experiment that has been repeated thousands of times in classrooms and by enthusiasts is a simple fact, the lower the wave length the more heat it has. It can't emit heat, it is the heat. An electron, a quark, gluon, neutrino, all other basic particles can't be hot, that is not the quality of the energy that they posses, an atom essentially will never be hot even if it releases a particle that has a lot of heat.

In the paper "heat, quantum mechanics, and information" it was suggested that the first particle released in any electro magnetic phenomenon must be heat, the logic being how else can we can this chain reaction, as explained above all photons must have some heat and Herschel in a way proves this correct. The lower the frequency the easier it is to be absorbed.

As high heat particles need least energy to be released, they will not have the amount of energy that is traditionally associated with photon energy, $\mathrm{E}=\mathrm{hf}$, but will have a higher $\mathrm{F}-\mathrm{hf}$ value as that energy would not be wasted in their release.

What are considered traditionally high energy particles will have more energy used (if one merely looks at things from what is information, humans are just an information package, there really is no such thing as wasted energy. What is wasted from our point of view might not be wasted from the rocks point of view.) in their release and are thus left with less heat, but that heat was transferred to what I term penetrating energy because I don't know what else to call it. The energy has not disappeared it has manifested itself differently.

When we look at black body radiation however one could say that is the opposite of reality, in reality we know red is hotter than blue. But with blackbody radiation we see that when we see blue we believe it is hotter. That is what is considered the illusion of black body radiation. But because a blackbody is supposed to absorb all electromagnetic activity, when we see radiation in the visible light and we see blue, it is not because blue light is hotter, it is because more energy has been used to produce those higher frequency photons, it takes more energy to create blue light than red light such that appears blue is hotter but when that blue light reaches us, it is actually cooler because it has taken more energy to create and we can see this clearly in black body radiation. It represents hotter at the origin because it took more energy to create, that energy being heat, but we are seeing its arrival, it has manifested more of that energy into its creation, its release. 


\subsection{Naturally occurring radiation}

${ }^{8}$ Maria Curie studied radioactivity to an intense level, finding that it occurs in several types of material naturally. X-rays and gamma radiation that occur naturally appear to be a different process as one can ask here we have release of highly ionizing radiation but there is no energy being put into the process it just seems to be only radiated out. We are dealing with unstable material that is not just changing phase but changing the internal structure, bonds are being broken at the atomic level that requires a lot of energy to be released and this energy is used to release electromagnetic activity. As the energy is so much the phenomenon that comes out is not hot but penetrating all the other quality of energy it can have been used up for its release. The energy quality of the electromagnetic phenomenon depends on how much energy is used to release it.

\section{Heat the Atmosphere and Terraforming}

We enjoy big experiments, and true without investigation in the last 200 years we would not understand wavelengths let alone frequencies and energy of a photon. But having understood these things the greatest experiment is all around us, the earths atmosphere, but it would be a meaningless experiment if we did not understand frequencies, wavelengths, and energy concerning the electromagnetic spectrum.

This paper has asserted that all photons have the same amount of energy, it takes energy to boil water and we know from Herschel's experiment that in the color spectrum, the visible light spectrum, longer wave lengths are hotter than the shorter wave lengths. However, even with all this evidence we still get for example the ${ }^{9}$ North Carolina Climate Office amongst many others stating, "Earth's radiation is emitted as longwave because longwave radiation contains a smaller amount of energy." Hopefully this paper will change such attitudes.

This is the time to keep things simple because if we can keep it simple we will have a serious question to answer around the temperature of the earth given the reality of electromagnetic activity around the earths atmosphere. The atmosphere of the earth.is like a giant collider but for photons, it is free, costs nothing, the costs rest merely on studying the results.

Figure 8 has some great importance to this paper in that it is part of what is interesting in what makes this final stage of the paper and the relationships that we must understand, simple, but allowing our imagination to flow but at the same time remembering reality and understanding that reality allows a certain order. Every relationship results in a loss of freedom, understanding the relationships in the atmosphere will allow us to greatly understand heat. 


\section{Figure 8 : Atmospheric electromagnetic transmittance or opacity}

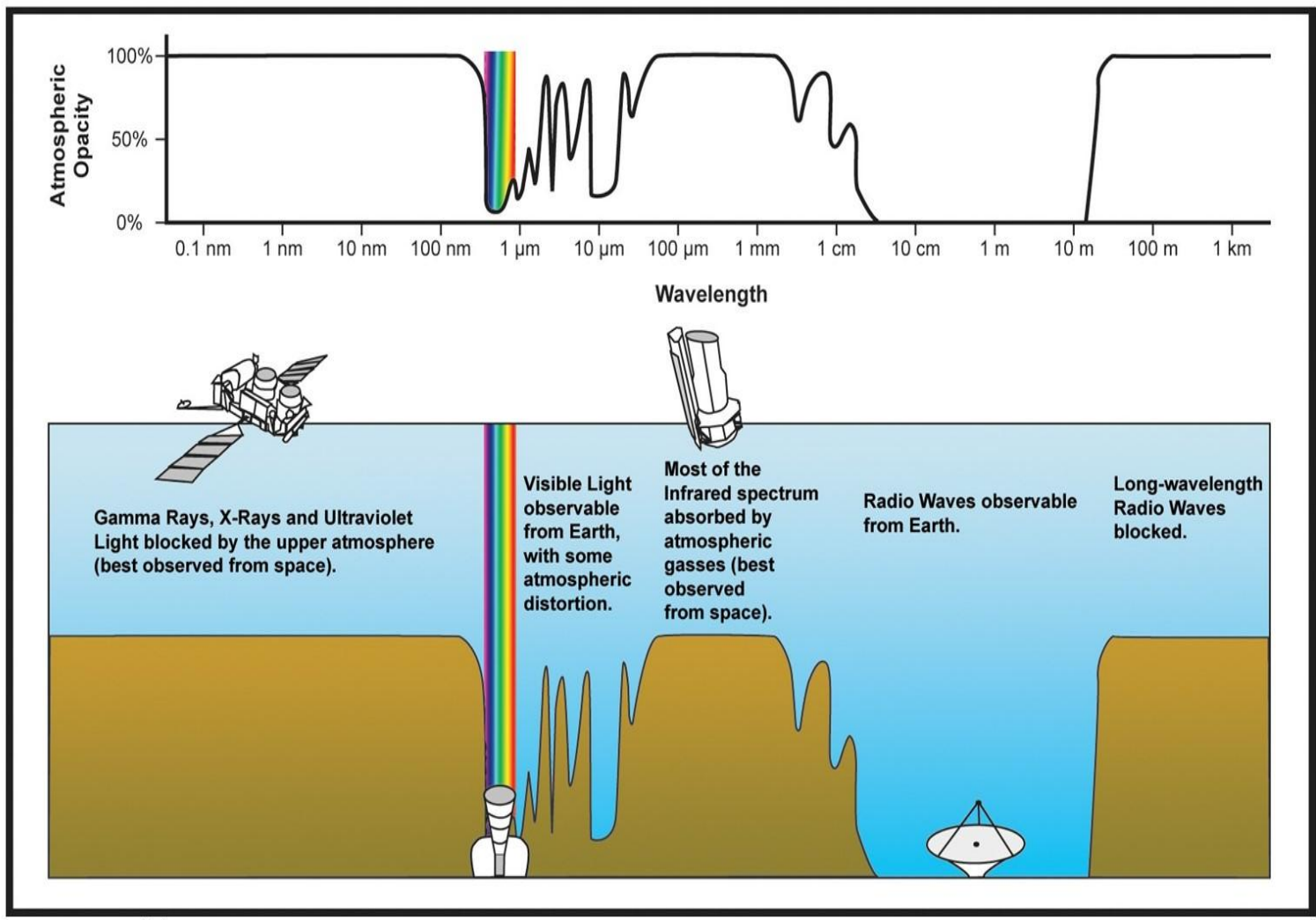

source: wikicommons

Figure 8 basically shows the atmospheric window that allows certain types of radiation to enter the atmosphere. Starting from the left, the shortest wavelengths like gamma, $x$ rays and most ultraviolet light is blocked by the atmosphere, ozone and oxygen. Visible light comes through the atmospheric window, it is not blocked that is why we are able to see. Some infrared is light in but most of it is blocked as well as microwaves by water and carbon dioxide and particles in the atmosphere. Then we have the radio window, however waves that are longer than 10 meters are completely blocked by electrical charges in the atmosphere. The anomaly it seems is the radio wave window, why is the earth not hotter?

\subsection{The Photonic Cycle}

Figure 8 though important shall not be discussed in detail. Such detail is all over the internet. A quick lesson can be learnt from the likes of the videos made by ${ }^{10}$ Jennifer Pontius, ${ }^{11}$ Jon Bergmann, and ${ }^{12}$ Elisabeth Snipes Spring amongst many other good videos. One gets a basic understanding that most radiation does not affect us because of the atmosphere. Electromagnetic phenomenon is absorbed or scattered and when absorbed it is usually radiated as a lower frequency photon and this gives us the idea of the photonic cycle as illustrated in figure 9 . 


\section{Figure 9: photonic cycle}

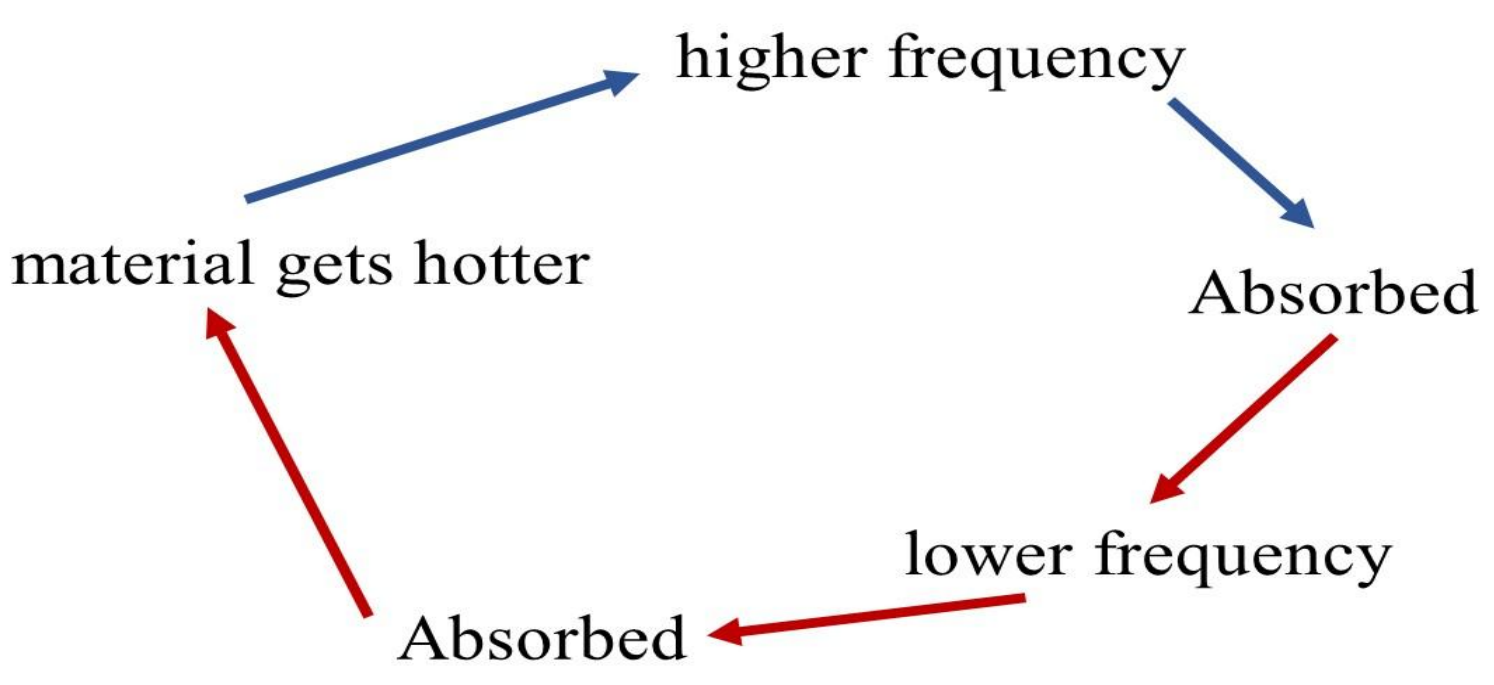

The photonic cycle covers complex information. Things prefer to be if possible, shown in the most basic of newtons laws that if something is moving it will not change direction until acted upon. It seems the universe wants to live, and to live it can not be at absolute zero, there is no activity. It has been around for billions of years but life is a recent phenomenon, but life is also just information, protons and neutrons, and electrons. But life in many instances creates its own heat. Mammals rely on recycling heat that plants absorbed and are a reactor keeping warm without any sunlight. Humans go further still creating fire, smelting iron, the photonic cycle getting a chance, the universe by very tiny margins moving away from ever getting to absolute zero, because intelligent life can use stored energy, in 250000 years it has reached space, out of a couple of billion years, the universe has quickly learnt to create fire and keep the ideal photonic cycle needed if indeed the natural cycle of the universe is that it is cooling, the universe is alive when there is photonic activity the universe is active, adapting Intelligent life can eventually adapt to a universe without suns, exploiting the information around it to keep the universe active, to get to this stage of keeping the universe going intelligent life can't destroy itself, all a learning process of information, seemingly everything connected all adapting to keep the universe going. The electromagnetic spectrum has provided the technology such that there is always hope that it can remain active, the longest of waves containing the most heat energy, and these long waves theoretically begin with the tshison, the longest of the waves theoretically possible.

The photonic cycle is just the flow of energy needed to sustain intelligent life into perpetuity as it would be impossible for the universe to die, merely continuous recycling of energy.

\subsection{The Radio Wave window}

Before being interrupted by the photonic cycle, this paper asked the question why the earth is not hotter given the radio wave window. When we are done with understanding the radio wave window our very understanding of the circumstellar habitable zone must change. And given the reality of the paragraph above, the habitable zone should even increase further.

It is a fairly simple affair why the earth is not hotter and the very evidence has been in front of us for over a century. We shall look at 2 waves A and B as depicted in figure 10. 


\section{Figure 10: Wave A and B}
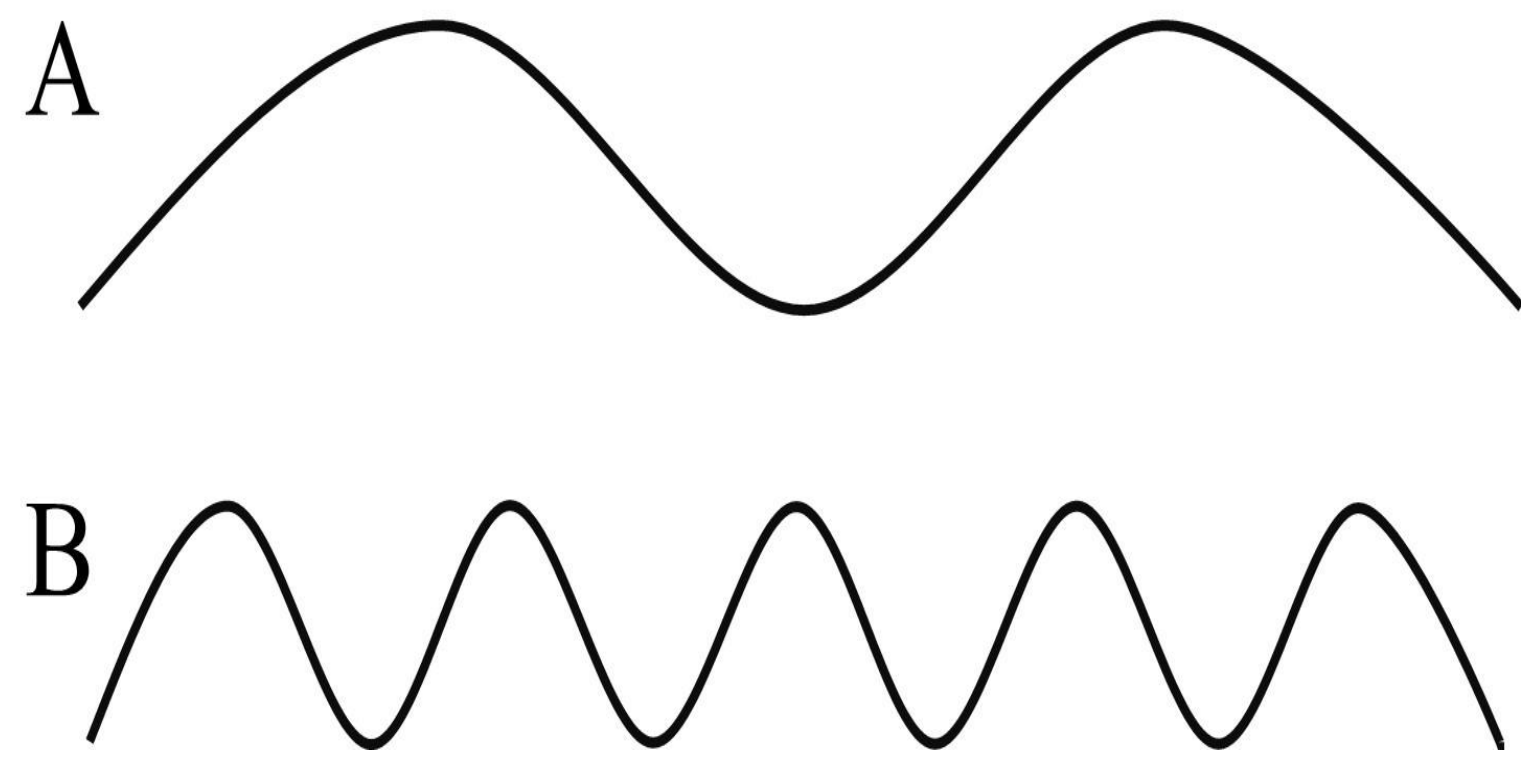

In figure 10 we see two waves, $A$ has a frequency of 2 and $B$ has a frequency of 5 . It has been accepted that energy of the wave is hf, plancks constant multiplied by the frequency in that regards we say that wave $B$ has more energy than wave $A$. And this fits with our everyday reality and all the visual reality of the description of a wave as depicted in figure 10. This is even further reinforced when we look at figure 10.1. 


\section{Figure 10.1: Barrier}

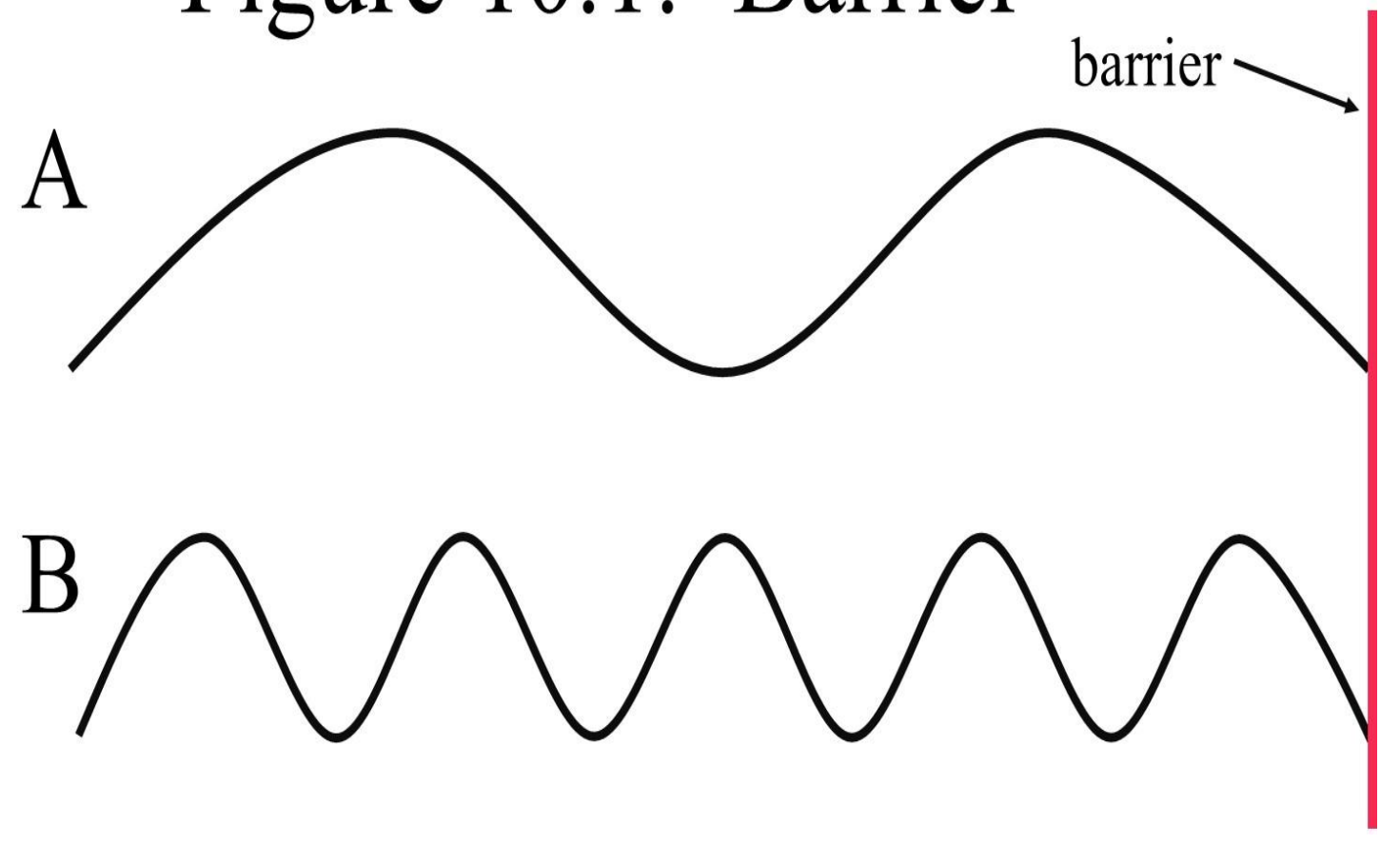

In figure 10.1 we have a barrier, in certain time, $t$, wave $A$ has 2 crests of the wave hitting the barrier whilst $B$ has 5 crests of the wave hitting it. The basic photon is the same all that is differentiating the photons is the frequency giving them different classifications. This is the obvious description from looking at illustrations, but we know that infrared is hotter than ultra violet even though ultraviolet has more energy, obviously just a type of energy. What we can see from figure 10.1 considering the differences between the waves, at the barrier where figure $A$ hits there will be less penetration but greater heat, and where $B$ hits the barrier there will be less heat but greater penetration and $B$ will be delivering energy at 5 times the rate of $A$.

At the same time however, A has delivered more heat than B by the same amount, not easily comprehended because one can not see it visually as when one draws a wave, but the heat is there. The heat is thus delivered more slowly but delivered none the less. We understand that all photons travel at the same speed in a vacuum, at that speed is c. We thus must look at speed from a photons view, and that speed has to do with frequency.

Imagine the photons as trains, the more loaded the train the slower it will go, but it is loaded with information. The less loaded the train the faster it will go, this is what the frequency shows us with regards to the electromagnetic spectrum. Thus ultraviolet light is like a train with less of a load than infrared and is thus going faster, this speed being measured by the frequency of the wave. When it hits a barrier, it penetrates more but still delivers less load. Infrared when it hits the barrier is slower, but it delivers a greater load, this load is heat. It is a terrible analogy the train, but it is the best one.

We can now understand why it seems earths design allows radio waves up to a certain point and this does not adversely affect life on earth, the temperature is not higher. It is the slowness of the radio waves given the frequency of microwaves right up to gamma are much quicker, but this speed must be understood in frequency because all the electromagnetic waves when merely looking at the 3-dimensional space and time, all the waves are moving at the same speed. Traveling faster does not mean having more energy, it just means having more of a different type of energy. When the photon is absorbed and released as lower frequency, it is as if it has picked up a load. 
The radio wave window allows this certain bands of radio waves in, they do not interfere with much, large particles and molecules do not affect them. These waves are so slow they are overwhelmed by the information delivered by higher frequency windows. Take infrared with a frequency of 10um and ultraviolet light with a frequency of $10 \mathrm{~nm}$. This means the micro wave we are talking of is 10 micro meters long and the ultraviolet light is 10 nano meters or ultraviolet light has a frequency of 10000 times greater than infrared. That means for every once that infrared light hits the barrier illustrated in figure 10.1, ultraviolet light will hit it 10000 times, this is the main reason why the shade is cooler than the separated colors in Herschel's experiment.

The earth is obviously having the right parameters for us to exist, and if where to exist on another planet we would merely need to mimic the conditions on earth. As the shade is cooler it means that there is far more ultraviolet activity in terms of influence than the rest of the atmospheric window combined in terms of heat. The atmospheric window allows radio waves up to 10 meters, ultraviolet light has a frequency 10 billion times that of these radio waves, anything more than this is blocked by electrical charges in the atmosphere. That is why we are not boiling over, the photonic balance and mixture as explained for figure 4 , the quality of heat.

\section{3 photonic mist}

When we look at a diagram released by ${ }^{13} \mathrm{EMF}$ Portal, we realize that humans are creating artificially very long waves that otherwise would not be present, and we know that it is a photon that is heat and nothing else. Humans are creating waves as long as 10000 kilometers, a kind of wave that would be naturally blocked by the earths atmosphere. Commercial radio waves can be as long as 100 kilometers, they have a lot of heat energy. It would thus be unfair to not look at the composition of the atmosphere in terms of quality of photon presence to fully understand climate change. Every cellphone signal could potentially be responsible for increasing the heat in the atmosphere. These waves are blocked from coming in, they will certainly be blocked from the same electrical charges from leaving.

The photonic mist is man made photons in the atmosphere. They must contribute to global warming if such an event is taking place.

\section{4. circumstellar habitable zone}

The circumstellar habitable zone is the zone where we expect to find ready made planets for life like our own

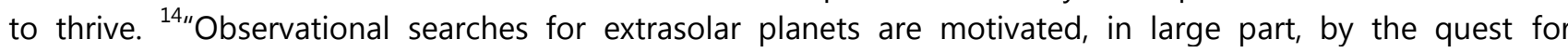
astronomical environments with physical and chemical conditions supportive of life. The criterion most commonly adopted to define such "habitable" environments is the presence of water in liquid phase."

With our understanding of heat this habitable zone increases in size. With the right photon mixture in the atmosphere any planet with an atmosphere potentially can have environments with liquid water. What is important is how the atmosphere captures and releases photons. A terrestrial planet with an atmosphere the distance equal to that of Neptune from the sun could theoretically have temperatures that allow liquid water because of how the electromagnetic spectrum interacts with the atmosphere.

Given the photonic cycle we understand that given the importance of the photon balance, given time even a planet like Venus can be settled. Not forgetting the full meaning of the photonic cycle, that the universe will still be able to create heat, the life sustainer, even without a sun, the early evidence of this is intelligent life that can manipulate fire and learn. Fire comes from stored energy and it is just being recycled.

Understanding our own planet, we know to reduce the temperature of the atmosphere of Venus. Understanding the atmosphere of Venus, we understand we must clean up. It must be cleaned up such the photonic content is reduced to desirable levels. At desirable levels, being closer to the sun, and after a good clean up, technology that would have cleaned up the earths carbon dioxide if that is what is truly necessary. The atmosphere of Venus will have to be manipulated in such a manner that the photonic content eventually allows for liquid water. Venus would be much easier than a terrestrial planet whose atmosphere is dominated 
by say methane. Venus has atmosphere dominated by carbon dioxide, something we have technology to break down. Then because of the proximity of Venus to the sun compared to that of Earth's, the atmospheric photonic content can not be the same, Venus will need more ultraviolet to cool the planet and less infrared window and radio wave window given reality.

A planet with correct atmospheric condition but merely needing heat because it is too far from any star, in time given the full implications of the photonic circle, intelligent life to prosper and sustain itself in this universe will find a way to introduce heat so that life can sustain itself there and utilize and sustain the photonic cycle. What is important is that intelligent life gives itself some guidelines such that it does not destroy itself, all reached by some sort of compromising system.

\section{Conclusions}

Besides understanding that heat is part of the energy of a photon, all photons, some having more than others, we understand the quality of a photon's energy. A photon just does not have one type of energy, hf, but also $\mp$ - hf, $\mp$ - hf being the expression for heat. If we could get a scale that measure the number of photons in an atmosphere and type, it could calculate the temperature more accurately than any thermometer known, as it would be acting as a scale. This thermometer would not just give the quantity, the total temperature but also the quality of heat, which part of the electromagnetic spectrum is responsible for the heat.

The concept of the thermometer falls in line with what the ${ }^{15}$ University of Wisconsin desires, progress. Their Atmospheric and Oceanic Sciences has as one of its primary mandates in understanding relationships between atmospheric phenomenon, the "design and fabrication of satellite, aircraft, and ground-based instruments. Scientists in the department and their collaborators at the University and elsewhere are particularly active in developing new instruments for remotes sensing our environment."

The thermometer of course is right up the ally in new instruments. But for it to be calibrated properly we need to understand how much each photon gives out in heat. If only we could count the number of photons in each color band of Herschel's experiment. If only it was that easy, simple division and we would know how much tshisa each photon has, tshisa being unit of photonic heat. If not properly calibrated, the tool has no use as a thermometer, it will not be able to tell us the differences in quality between two areas with a similar $32^{\circ} \mathrm{C}$ thousands of kilometers apart.

As heat comes from photons, it is important to understand the effects of the photonic mist on the environment. The photonic mist are photons artificially created by human beings like our cell phone technology, tv and radio stations. One could easily call the photonic mist the electromagnetic mist. But understanding the photonic cycle we understand that is our commercial source of energy, true we use it for communication, but that is only recently in human evolution.

We understand that with massless particles it is about the quality of the energy. As we progress we will learn, exploit the different. It is possible there are better methods of communications besides the photon which when understanding reality of photonic cycle was merely to keep the universe energised, and seemingly to allow intelligent life to understand what it is doing, but some intelligent life use infrared, beyond the humans capabilities, to that creature, infrared is invisible light. Massless neutrino go through the earth, learning to exploit them means satellites not needed as they will go through the earth, that is quality of their energy. Being able to go through the earth means the neutrino has different quality of knowledge not more or less than a photon or other massless particles.

What we understand about evolution is that it is a process about adapting to the environment.

The photonic cycle is the sustenance of the universe, the continual activity of the electromagnetic spectrum. This is one possibility but the only one viable for life, it will be the most difficult thing intelligent achieves, but once they get there it will be like nothing, each generation adding a block to the knowledge base. 


\section{Funding Statement}

This research is self funded

\section{Acknowledgments}

I acknowledge the universe for giving me this opportunity

\section{References}

[1] The Physics Classrom, What is Heat? Retrieved 20 Oct 2018

[2] Khumalo, B. (2016) Why Economics Is a Science: Information, the Grand Unifier, https://www.scirp.org/journal/PaperInformation.aspx?PaperID=63721

[3] Khan Academy, Heat and temperature, https://www.khanacademy.org/science/chemistry/thermodynamicschemistry/internal-energy-sal/a/heat retrieved 28 October 2018

[4] Khumalo, B. (2015). Heat, quantum mechanics and information. JOURNAL OF ADVANCES IN PHYSICS, 10(2), 2692-2695. https://doi.org/10.24297/jap.v10i2.1328

[5] NASA, The Electromagnetic Spectrum, https://imagine.gsfc.nasa.gov/science/toolbox/emspectrum1.html retrieved 30 October 2018

[6] Coolcosmos, Official website, http://coolcosmos.ipac.caltech.edu/ retrieved 30 Oct 2018

[7] Coolcosmos, An Wxample of the Herschel Infrared Experiment,

http://coolcosmos.ipac.caltech.edu/cosmic_classroom/classroom_activities/herschel_example.html retrieved 30 Oct 2018

[8] Bagley, M. (2013) Marie Curie: Facts \& Biography, https://www.livescience.com/38907-marie-curie-factsbiography.html

[9] North Carolina Climate Office, Longwave and Shortwave Radiation: retrieved Nov 32018

[10] Pontius, J. (2017) atmospheric interactions, https://www.youtube.com/watch?v=4NWgEfbNzho

[11] Bergmann, J. (2010) Absorption of Electromagnetic Waves in the Atmosphere, https://www.youtube.com/watch?v=EzV5JBBY7GE

[12] Spring, E.S (2013) Ch02S Atmospheric Windows, https://www.youtube.com/watch?v=dykqL1xGG_A

[13] EMF-Portal, Electromagnetic spectrum, https://www.emf-

portal.org/en/cms/page2/home/technology/general/electromagnetic-spectrum date retrieved: 6 Nov 2018

"[14] Vladilo,G. Murante, G. Silva, L. Provenzale, A. Ferri, G. Ragazzini, G. The habitable zone of Earth-like planets http://adlibitum.oats.inaf.it/astrobiology/planhab/docs/pressHab.pdf with different levels of atmospheric pressure" retrieved 30 September 2018

[15] University of Wisconsin, Radiation and Remote Sensing, https://aos.wisc.edu/research/radiation/ date retrieved 8 Nov 2018 